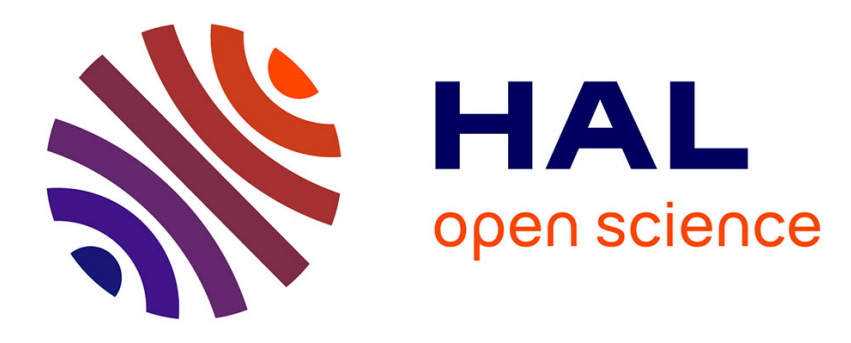

\title{
Une histoire transnationale des idées juridiques ?
}

Jean-Louis Halpérin

\section{To cite this version:}

Jean-Louis Halpérin. Une histoire transnationale des idées juridiques ?. Clio@Thémis. Revue électronique d'histoire du droit, 2018, 14, 10.35562/cliothemis.751 . halshs-03279485

\section{HAL Id: halshs-03279485 \\ https://shs.hal.science/halshs-03279485}

Submitted on 6 Jul 2021

HAL is a multi-disciplinary open access archive for the deposit and dissemination of scientific research documents, whether they are published or not. The documents may come from teaching and research institutions in France or abroad, or from public or private research centers.
L'archive ouverte pluridisciplinaire HAL, est destinée au dépôt et à la diffusion de documents scientifiques de niveau recherche, publiés ou non, émanant des établissements d'enseignement et de recherche français ou étrangers, des laboratoires publics ou privés. 


\section{Une histoire transnationale des idées juridiques?}

Jean-Louis Halpérin

(c) https://publications-prairial.fr/cliothemis/index.php?id=751

DOI : 10.35562/cliothemis.751

Référence électronique

Jean-Louis Halpérin, "Une histoire transnationale des idées juridiques? ?, Clio@Themis [En ligne], 14 | 2018, mis en ligne le 19 avril 2021, consulté le 24 avril 2021. URL : https://publications-prairial.fr/cliothemis/index.php?id=751

Droits d'auteur

CC BY-NC-SA 


\title{
Une histoire transnationale des idées juridiques?
}

\author{
Jean-Louis Halpérin
}

\section{PLAN}

I. L'histoire transnationale des idées juridiques : dans quelle mesure?

II. L'histoire transnationale des idées juridiques : avec quels moyens?

III. L'histoire transnationale des idées juridiques : à quelles fins?

\section{TEXTE}

1 L'essor de l'histoire transnationale, globale ou connectée ne peut manquer d'avoir un impact sur l'étude diachronique des idées juridiques, dans la mesure où elle a placé les «transferts culturels » ou la circulation des idées aux premiers rangs de ses objets ${ }^{1}$. Pour autant, la réflexion des historien(ne)s du droit, encore limitée sur l'histoire transnationale des normes juridiques ${ }^{2}$, ne s'est guère portée sur les possibilités et les difficultés d'une histoire transnationale des idées juridiques. L'on serait tenté de dire que cette question se pose depuis longtemps et ne paraît pas avoir suscité des problèmes majeurs aux spécialistes d'histoire des idées juridiques, comme à ceux d'une discipline voisine, plus anciennement identifiée notamment en France, l'histoire des idées politiques. Cela fait des générations, par exemple, que les auteurs français qui se livrent à l'histoire des idées, dans ses rapports avec le droit, étudient Platon et Aristote, Bracton et Marsile de Padoue, Machiavel, Grotius, Hobbes, Kant, Hegel, Savigny, Holmes ou Kelsen aux côtés de Bodin, Domat, Montesquieu, Hauriou ou Duguit. Les ouvrages de Michel Villey sur l'histoire de la philosophie du droit ne se sont jamais inscrits dans un cadre national ${ }^{3}$. Personne n'oserait, du moins aujourd'hui, proposer comme Roger Daval l'avait fait dans un Que-sais-je ? des années 1950/1960 une « histoire des idées en France » se limitant à une « filière » d'idées inscrites dans un seul contexte national, lui-même identifié par la nationalité des auteurs considérés ${ }^{4}$. Aucun pays ne peut prétendre avoir le monopole des idées ou d'une catégorie d'idées. 
2 Nul doute que les idées juridiques, ou ce que Rodolfo Sacco a appelé en 1991 des «formants juridiques » pour qualifier des ensembles plus complexes de concepts présents dans la littérature juridique, circulent par-delà les frontières ${ }^{5}$. Ce qui est évident pour le domaine politique, où les idées font précisément l'objet d'un débat public impliquant des auteurs de nationalités variées, ainsi que des échanges élargis entre des «savants » et des groupes sociaux réunissant des « lecteurs » ou non-spécialistes, l'est-il autant pour les idées juridiques ? Celles-ci font, en effet, partie des «idées ésotériques », selon le vocabulaire de L'histoire des idées de Marc Angenot ${ }^{6}$, qui circulent dans des cercles plus restreints, la plupart du temps réservés aux seuls juristes. Au premier abord, l'on peut dire que la circulation de ce type d'idées «savantes » est facilitée à l'intérieur de communautés épistémiques : les mathématiciens, les économistes, les philosophes n'ont pas de difficulté particulière pour communiquer à travers le monde et pour utiliser, depuis longtemps, des textes venus d'autres pays et d'autres langues. De plus, la frontière est loin d'être étanche entre idées juridiques et idées politiques, ce qui devrait faciliter la circulation de la pensée juridique par-delà les frontières. La question de la langue nous montre toutefois, avec les exemples empruntés à d'autres discours savants, qu'il est plus facile aujourd'hui aux scientifiques de communiquer en utilisant la plupart du temps la langue anglaise. Cela veut-il dire que la circulation des idées juridiques, facile à l'époque où les ouvrages juridiques étaient écrits en latin, est devenue difficile avec l'avènement des langues vernaculaires ? Les discours juridiques sont en tout cas liés plus ou moins étroitement à des droits positifs qui se sont constitués, au fur et à mesure de l'histoire moderne et contemporaine, sur des bases nationales et sont de la sorte séparés par des frontières. Il faut donc s'interroger sur l'espace propre à une (ou plusieurs) histoire(s) transnationale(s) des idées (I), avant de voir les méthodes à développer dans ce type d'études (II) et les objectifs heuristiques poursuivis par les démarches transnationales (III). 


\section{L'histoire transnationale des idées juridiques : dans quelle me- sure?}

Nous reconnaissons tous, en tant qu'historien(ne)s du droit, que nos recherches impliquent de plus en plus un point de vue « européen » et international qui n'était pas celui de nos prédécesseurs d'avant la Seconde Guerre mondiale. Nous travaillons dans une discipline qui a été établie dans un cadre universitaire national, en tant qu'histoire du droit national et de ses racines avec même des accents nationalistes qui, sans avoir la même prégnance qu'autrefois, se sont transformés en une légitime fierté pour «notre » histoire. Nous sommes bien évidemment, par notre éducation et par notre environnement, plus familier de l'histoire du droit de notre pays que de celle des pays voisins, a fortiori de celle de pays éloignés. Nous devons tenir compte de cet obstacle quand nous examinons l'histoire des transferts juridiques ou de la circulation des énoncés normatifs. Or, il semble que les efforts que nous déployons pour nous intéresser à l'histoire du droit d'autres pays, et notamment à l'histoire des droits européens, ont besoin en quelque sorte d'être redoublés ou amplifiés quand nous abordons l'histoire des idées juridiques, c'est-à-dire un domaine qui concerne bien sûr les phénomènes juridiques, mais s'insère aussi dans une histoire intellectuelle ou culturelle beaucoup plus large.

4 Les problématiques de l'histoire des idées, à commencer par le statut même de cette discipline ou des démarches qu'elle prétend réunir, débordent très largement le domaine juridique et intéressent une très vaste littérature historique, politique et sociologique. Non seulement il est très difficile à un seul chercheur de se tenir au courant de l'immense production de cette histoire des idées, mais les habitus créés par notre éducation et entretenus par les cadres universitaires nationaux ne nous rendent pas directement accessibles les productions marquantes qui se font en dehors de nos frontières en matière d'histoire des idées. Si l'on prend l'exemple de la France, où les frontières sont particulièrement étanches entre les facultés de droit et les autres disciplines universitaires, il n'est pas surprenant que les historiens du droit soient plus familiers des idées connues dans le paysage 
intellectuel français que des livres, pourtant riches et susceptibles de traiter des mêmes sujets, produits dans d'autres pays. Les spécialistes d'histoire du droit français à l'époque contemporaine ont souvent pris des années de retard pour lire des ouvrages d'universitaires de langue anglaise qui traitaient des auteurs de la doctrine française ${ }^{7}$. Ce phénomène est aggravé pour tout ce qui concerne la méthodologie même de l'histoire des idées : quel spécialiste d'histoire du droit peut prétendre maîtriser la littérature internationale sur les mouvements d'idées socialistes et communistes à travers le monde, alors que ces mouvements ont pu influencer des juristes au $\mathrm{xIX}^{\mathrm{e}}$ et au $\mathrm{xx}^{\mathrm{e}}$ siècles?

Il ne faut pas sous-estimer la prégnance des tropismes et des cadres nationaux encore aujourd'hui dans l'histoire des idées juridiques. Les Allemands font de l'histoire du droit privé ou public en Allemagne, les Italiens de l'histoire de la science juridique italienne, les Britanniques de l'histoire des auteurs de common law, les Français de l'histoire de la culture juridique française ${ }^{8}$. Et nous n'avons pas toujours le réflexe consistant à questionner ce cadre national et à nous demander s'il a vraiment existé une «pensée juridique » allemande, italienne ou française. Pour aborder l'histoire transnationale des idées, il faut approfondir notre compréhension de ce que signifie la nationalisation du droit et des idées juridiques, il faut s'interroger sur le perpétuel travail de réinvention des identités culturelles et nationales. S'il y a du sens à rattacher un auteur, et donc indirectement ses idées, au pays dans lequel il a travaillé et publié (qui ne correspond pas toujours à sa nationalité d'origine), il convient de prendre quelques distances avec une étiquette nationale, de ne pas conclure hâtivement de la production d'un juriste français, allemand, italien ou britannique à l'existence d'une idée «française », « allemande », « italienne » ou « allemande ». Dans le temps comme dans l'espace, un certain nombre de caveat paraissent nécessaires pour déterminer ce qui relève plutôt de l'histoire nationale des idées et ce que peut apporter une perspective transnationale dans l'histoire des idées.

6 Le premier caveat repose sur l'idée qu'il n'y a pas de «transnational » tant qu'il n'y pas de nations constituées en tant que telles et de phénomènes que l'on peut qualifier de «nationalisme juridique ». Pour les périodes les plus anciennes de l'Antiquité et du Moyen Âge, il y a bien sûr d'importants phénomènes de circulation des idées juridiques : il suffit de songer aux relations entre la jurisprudence romaine et la 
philosophie grecque, aux débats religieux qui ont pu impliquer au Moyen Âge des savants chrétiens, romains ou orthodoxes, juifs et musulmans et bien sûr à la diffusion à partir de l'Italie de la littérature des glossateurs et commentateurs, utilisée jusqu'à l'époque moderne par les juristes de toute l'Europe qui écrivaient en latin. Mais y a-t-il beaucoup de sens à parler d'histoire transnationale quand les juristes ne sont pas, pour la plupart, attachés à un État (à supposer que ce concept ait un sens pour le plus grand nombre des configurations historiques de ces périodes) et où beaucoup d'entre eux voyagent, enseignent en plusieurs lieux et voient leurs manuscrits latins essaimer à travers toute l'Europe ? La problématique transnationale suppose la consolidation des frontières entre des territoires et des populations liés à des États modernes, ainsi que le développement de littératures en langue vulgaire qui tendent à isoler relativement différents corpus de productions juridiques. L'on sait que l'expression «droit français » apparaît au xvi ${ }^{\mathrm{e}}$ siècle quand une doctrine aux accents nationalistes, partie du mos gallicus pour arriver à l'exaltation des coutumes comme «vrai droit civil », soutient la construction de l'État royal ${ }^{9}$. Lépoque moderne est caractérisée en Europe par la coexistence de littératures juridiques en langue vulgaire et le maintien de publications en latin qui sont plus nombreuses dans les espaces où la structuration de l'État national est moins avancée. La France a connu de manière particulièrement précoce, à partir du début du xvII ${ }^{\mathrm{e}}$ siècle, l'essor des livres juridiques en français, ce qui n'est pas sans rapport avec le renforcement de l'absolutisme et a conduit à l'établissement des professeurs de droit français dans les facultés en 1679. L'Angleterre, où la Réforme a renforcé l'idée que le common law était un don de Dieu au peuple anglais ${ }^{10}$, a suivi aussi une voie qui permet de parler d'une science nationale du droit. Les exemples du Saint-Empire et de l'espace italien sont plus complexes avec la coexistence d'un fort maintien d'une littérature juridique en latin et de l'apparition de livres de droit en allemand ou en italien.

Lusage, plutôt relâché, que nous faisons du terme d'« école juridique » devrait nous amener à réfléchir davantage sur les implications du renforcement à partir $\mathrm{du} \mathrm{xvII}^{\mathrm{e}}$ siècle des frontières linguistiques et nationales. Ce n'est pas un hasard si l'École du droit naturel moderne a trouvé son essor dans un espace centré sur les Pays-Bas, le Saint-Empire et les pays scandinaves. Outre ses liens avec le pro- 
testantisme, elle a profité du maintien dans ces territoires du latin comme principale langue du droit. Si Grotius et Pufendorf ont été traduits en français, ces traductions ont pris du temps, ce qui permet d'expliquer les effets-retard de l'influence de leurs travaux en France. Hobbes ou Locke ont été pour leur part traduits en latin et en français, ce qui nous rappelle que leurs œuvres originales en anglais n'ont pas été tout de suite accessibles à un public non anglophone. Le phénomène bien connu de l'essor de la littérature des Institutes de droit national ${ }^{11}$, pour une part en latin, pour une autre part en langue vulgaire, confirme la transition qui s'est opérée entre un espace juridique latin et une véritable circulation transnationale. Avec les codifications de la fin du xviII ${ }^{\mathrm{e}}$ siècle et du début du $\mathrm{xIx}^{\mathrm{e}}$ siècle, la nationalisation du droit s'accompagne de réformes étatiques des études juridiques (particulièrement en France et en Autriche) qui provoquent la formation d'écoles de droit dans des cadres strictement nationaux, c'est-à-dire où l'on enseigne presque exclusivement le droit national dans la langue du pays. Dans les territoires de langue allemande, le choc provoqué par l'impérialisme français a également suscité une prise de conscience qui conduit, à travers des revues et des ouvrages en allemand, à la constitution d'un « champ juridique allemand » où les auteurs débattent notamment de la question de la codification. Cela donne son sens au qualificatif d'allemande donné à l'École historique du droit. S'il ne faut pas en conclure trop vite que des « écoles nationales » de droit existent désormais partout en Europe, les structures de l'enseignement et du droit positif font que les juristes français parlent presque uniquement de droit français, les juristes allemands de droit allemand, les juristes italiens de droit italien. Si l'on prend le terme d'école dans un sens plus précis, supposant la présence de maîtres et de disciples partageant les mêmes idées et pouvant s'organiser pour les diffuser, le phénomène est encore plus ancré dans certaines traditions nationales. Le cas de l'Allemagne, où de telles écoles ont pu se constituer dans la seconde moitié $\mathrm{du} \mathrm{xIX}^{\mathrm{e}}$ siècle sur la base de séminaires des professeurs les plus réputés, ne doit pas être généralisé à d'autres pays, dont la France, pour lesquels de telles structures n'existaient pas.

Pour qu'il y ait une circulation transnationale des idées juridiques, il faut des frontières à franchir, il faut que des juristes s'intéressent à des livres produits dans d'autres pays, il faut que des idées d'abord 
liées à un ordre juridique particulier influencent des juristes relevant d'un autre ordre juridique. L'histoire transnationale des idées juridiques nous parait concerner les seules époques modernes et contemporaines et suppose une contextualisation constante dans les espaces politiques et linguistiques (on songe, par exemple, au recul de la connaissance du français entre les $\mathrm{xvIII}^{\mathrm{e}}-\mathrm{xIX}^{\mathrm{e}}$ siècles et le $\mathrm{xx}^{\mathrm{e}}$ siècle) qui déterminent les frontières à franchir pour la circulation des livres et des concepts qu'ils contiennent.

Un deuxième caveat consiste à tenir compte des configurations disciplinaires et des sujets dont discutent les juristes entre eux, le plus souvent dans un contexte national, pour nous en tenir au $\mathrm{xIx}^{\mathrm{e}}$ et au $\mathrm{xx}^{\mathrm{e}}$ siècle. L'on sait que le découpage des « disciplines » n'est pas, et n'a jamais été, le même dans la formation juridique d'un pays à l'autre. Beaucoup de controverses juridiques sont liées à l'interprétation de textes nationaux, notamment des codifications censées faire système, ou à la discussion de situations politiques propres à un État (par exemple, la question budgétaire en Prusse dans les années 1860 n'avait pas de raison d'intéresser particulièrement les Français). Il est ainsi des domaines qui sont plus ouverts à la pénétration d'idées étrangères que d'autres. L'on pense aux nouvelles «spécialités »du $\mathrm{XIX}^{\mathrm{e}}$ siècle : le droit international (public ou privé), le droit du travail (du fait des décalages entre législation nationale et législations étrangères qui suscitaient l'intérêt de tous ceux qui voulaient faire «progresser » la législation ouvrière) ou l'histoire du droit (du fait de la circulation des travaux sur l'histoire du droit romain ou du droit germanique susceptibles d'intéresser les spécialistes de plusieurs pays ${ }^{12}$. Encore faut-il tenir compte, même dans ces domaines a priori susceptibles d'études comparées, de l'existence ou non de congrès ou d'associations à caractère transnational : c'est le cas pour le droit international public (Martti Koskenniemi l'a montré pour l'Institut de droit international ${ }^{13}$ ), pour le droit du travail (un auteur comme Paul Pic utilise beaucoup les travaux issus de congrès internationaux) ou pour la propriété intellectuelle (avec les conventions de droit uniforme de Berne et Paris). L'existence même de ces congrès a, bien sûr, facilité la circulation transnationale des idées juridiques.

10 En revanche, dans les domaines où ces rencontres entre juristes de plusieurs pays sont absentes ou plus restreintes, la communication n'a pu se faire qu'à distance par la circulation des livres ou des revues, 
ce qui a pu entraîner des délais dans la prise de connaissance d'idées exprimées à l'étranger. L'exemple du droit international privé, beaucoup plus dépendant de sources nationales, nous montre comment Bartin et Khan ont pu étudier parallèlement les conflits de qualification à la fin du xix ${ }^{\mathrm{e}}$ siècle en s'ignorant totalement ${ }^{14}$. Le domaine de la procédure civile est caractérisé par une circulation plutôt lente : ainsi la doctrine italienne autour de Chiovenda n'en fut diffusée que des décennies plus tard en France par Vizioz ${ }^{15}$. Si l'on prend le cas du droit pénal, la circulation des idées était difficile et donc très faible, jusqu'à la fin du xix ${ }^{\mathrm{e}}$ siècle, entre des pénalistes allemands (autour d'une vingtaine à chaque génération) travaillant sur l'élaboration de nouveaux codes et les pénalistes français (moins d'une dizaine par génération) se contentant de commenter la codification napoléonienne. Il faut attendre la fondation de l'Association internationale de droit pénal par Liszt, Hamel et Prins en 1889 (on remarquera d'ailleurs l'absence de pénaliste français parmi les fondateurs) pour que le débat, centré sur la réception, elle aussi partielle, des travaux de l'École positiviste italienne, prenne une ampleur transnationale. Des matières comme le diritto ecclesiastico et le Kirchenrecht n'ayant pas d'équivalent en France, les juristes français sont restés pour la plupart ignorants de la littérature italienne et allemande sur de tels sujets. De même, la faiblesse des connaissances philosophiques des juristes français les a probablement rendus imperméables, à la lecture d'Hegel, des néo-kantiens et donc de toute la littérature sur les normes juridiques (Thon, Binding, Bierling, Zitelmann, Bergbohm ont été pratiquement ignorés en France), puis (en dehors de Charles Eisenmann) à la compréhension de Kelsen (sur ce dernier, la lecture de ce qu'en écrit Hauriou est édifiante par l'accumulation des contresens $\left.!^{16}\right)$.

11 Il ne faut pas chercher du «transnational » partout dans l'histoire des idées juridiques à toutes les époques et dans tous les domaines de la «science juridique ». En prenant la mesure des effets de frontière et des obstacles à la circulation des idées, l'on doit distinguer les périodes et les objets pour mener à bien des analyses contextuelles, sans pour autant renoncer à des études centrées localement, tant la force des cadres nationaux est devenue prégnante à l'époque contemporaine. L'histoire coloniale doit faire, à notre avis, l'objet d'un traitement spécifique, en raison des processus d'imposition forcée 
des normes (et donc des idées juridiques) de la métropole. C'est aussi en s'appuyant sur les travaux déjà réalisés dans une perspective plus «nationale » qu'il est possible de développer à la fois des comparaisons et des formes d'histoire connectée. Tandis que les comparaisons s'appuient sur la juxtaposition et la confrontation d'histoires nationales pour mettre en valeur les ressemblances et les différences intéressant l'histoire des idées juridiques, les études d'histoire connectée s'attachent au cœur de la dimension transnationale : les circulations et les transferts d'idées qu'il faut identifier et analyser. Ne relève pas de l'histoire connectée l'étude des idées juridiques qui forment le fonds commun de la science du droit dans plusieurs pays, comme celles héritées du droit romain. C'est le transfert d'une idée, apparue d'abord dans un contexte national, dans un ou plusieurs autres pays qui constitue l'élément essentiel d'une perspective véritablement transnationale qui nécessite des moyens spécifiques.

\section{L'histoire transnationale des idées juridiques : avec quels moyens?}

12 Compte tenu des obstacles dressés par les frontières politiques, linguistiques et culturelles, il faut partir de l'hypothèse que la circulation des idées juridiques ne se présume pas, même si elle est toujours possible et envisageable. Une histoire transnationale des idées doit «pister » le passage de « concepts » (et de noms de concepts ${ }^{17}$ ) ou de théories juridiques d'un espace national à un autre. Ce travail d'enquête se mène principalement à travers les écrits témoignant de cette circulation d'un environnement à un autre : les textes rédigés dans un pays déterminé sont susceptibles de montrer qu'une idée venue de l'étranger est connue dans ce pays et de voir comment elle est comprise ou utilisée. L'histoire transnationale des idées juridiques se fait avec les méthodes de la bibliométrie, ce qui amène à une réflexion sur la connaissance des langues par les juristes, leurs lectures, la diffusion des livres et la naissance de mouvements intellectuels liés à des idées importées de l'étranger.

13 La question linguistique n'existe pas seulement pour nous, qui avons en règle générale plus de mal à lire et surtout à restituer dans leur 
environnement intellectuel les textes rédigés dans une langue étrangère, mais pour les auteurs du passé dont nous étudions les idées. Le fait qu'un Français d'aujourd'hui puisse lire Savigny et Jhering, Beccaria et Santi Romano, Bentham et Holmes, que ce soit dans des traductions ou dans le texte original, ne veut pas dire qu'il était aussi facile pour des juristes français du passé de connaître et d'aborder les ouvrages de ces auteurs. L'histoire des lectures des œuvres juridiques étrangères supposerait, dans l'idéal, une connaissance très pointue des lecteurs et de leurs connaissances linguistiques. Sur ces connaissances nous n'avons souvent que des indices, par exemple l'apprentissage de plusieurs langues étrangères par Daguesseau ${ }^{18}$ ou les faiblesses de Duguit concernant la compréhension de la langue allemande. Il est clair que l'immense majorité des auteurs européens du $\mathrm{XIX}^{\mathrm{e}}$ et $\mathrm{du} \mathrm{xx}^{\mathrm{e}}$ siècle n'ont pas accès à des ouvrages rédigés en arabe, en chinois ou en japonais qui n'ont pas été traduits dans d'autres langues. Au mieux, le fait que des juristes aient traduit eux-mêmes des textes étrangers ou traduit en langue étrangère des textes en langue maternelle attestent de compétences vérifiées dans le domaine linguistique. L'histoire transnationale des idées juridiques commence avec la diffusion de mots empruntés à d'autres littératures nationales et qui étaient peu ou pas usités dans les pays importateurs. Par exemple, le terme de «norme » acquiert une importance primordiale d'abord en Allemagne, dès le xvII ${ }^{\mathrm{e}}$ siècle ${ }^{19}$, puis surtout à partir des textes de Jhering, Thon et Binding ${ }^{20}$. « Norma » était pourtant connue par des juristes italiens de la moitié du xIX $^{\mathrm{e}}$ siècle $^{21}$, à une époque où l'emploi de cette terminologie est absent en France. Gény est un des premiers, sinon le premier, en France à utiliser les expressions de norme et de discipline normative, comme d'ordre juridique, toutes venues d'Allemagne, ce qui confirme sa très bonne connaissance de la littérature d'outre-Rhin ${ }^{22}$. Bien plus, il mobilise la notion de «positivisme juridique » en $1915^{23}$, alors que cette formulation complète (à ne pas confondre bien sûr avec les mentions du «droit positif ») était très récente en Allemagne ${ }^{24}$. Ce type d'enquête peut paraître pointilleuse, mais elle permet de tracer la circulation des noms de concepts et à travers elle la pénétration d'expressions connotées, devenues pour nous si familières que nous avons oublié leur ancienneté très relative. 
14 Cette histoire transnationale implique, ce que nous faisons davantage, des investigations précises sur les traductions, la présence des livres dans les bibliothèques et l'inventaire des citations empruntées à des auteurs étrangers. Ce que réalisent les médiévistes en suivant les pistes de tous les manuscrits juridiques à travers l'Europe, nous devons le faire également, en tenant compte des spécificités de chaque contexte, pour les époques moderne et contemporaine. Aux Temps modernes, où l'on ignore l'usage des notes avec des références précises (une pratique dont il faudrait aussi suivre les lents progrès au $\mathrm{xIX}^{\mathrm{e}}$ siècle), nous devons nous aider davantage de ce que les spécialistes de l'histoire du livre nous disent de la circulation, souvent interdite par le pouvoir, des livres de l'étranger ${ }^{25}$, comme des catalogues de bibliothèques privées qui ont été conservés ${ }^{26}$. Comme les auteurs anciens des $\mathrm{xVII}^{\mathrm{e}}$ et $\mathrm{xVIII}^{\mathrm{e}}$ siècle, et même ceux du xIX ${ }^{\mathrm{e}}$ siècle, n'avaient pas les mêmes pratiques que les nôtres en matière de références il y a bien des influences qui ont été tues ou restent mystérieuses. On s'interroge depuis des années sur la question de savoir si Domat avait lu Grotius et Hobbes ${ }^{27}$. La question est plus vaste : un auteur comme Domat ne cite pas ses sources doctrinales, quelles qu'elles soient. En partant d'une présomption d'ignorance des textes étrangers, plus difficilement accessibles, quelques indices peuvent être utilisés. Grotius et Hobbes ont vécu plusieurs années en France ${ }^{28}$. Les Jésuites ont été très vite intéressés par l'œuvre de Grotius ${ }^{29}$. L'hypothèse a été faite que Pascal et Nicole avaient connu les idées de Grotius et Hobbes, même si l'édition latine des œuvres de ce dernier date de 1668, après la mort de Pascal en $1662^{30}$. En sens inverse il faut attendre la première moitié du xvIII ${ }^{\mathrm{e}}$ siècle pour que Hobbes soit mieux diffusé en France ${ }^{31}$ et pour que la lecture de Grotius soit recommandée par Daguesseau. L'on ne peut exclure que Domat ait connu les idées de Grotius et de Hobbes (ou de l'un d'entre eux, la chronologie de la diffusion de leurs livres en France étant différente de celle des publications originales) et il reste, selon nous, à pister dans le texte de Domat ce qui pourrait relever de l'influence ou de la critique de ces auteurs « étrangers ». L'enquête reste ouverte sur d'éventuels transferts transnationaux.

15 Pour l'époque contemporaine, la prudence s'impose aussi. Alfons Bürge a montré comment la philosophie de Kant, mentionnée de manière très allusive par Portalis (et certainement ignorée par les autres 
codificateurs), avait pénétré dans les milieux juridiques français par les canaux étroits de quelques auteurs : le professeur de philosophie Charles Villers, le conseiller d'État de Gérando, le magistrat Renouard ${ }^{32}$. Appliquée à la première moitié du xIx ${ }^{\mathrm{e}}$ siècle, jusqu'aux célèbres traductions de Jules Barni sous le Second Empire, cette méthode rend sceptique sur une affirmation longtemps répétée : la prétendue influence de la morale kantienne sur les juristes français de la Troisième République. Combien de juristes français de la fin du $\mathrm{xIX}^{\mathrm{e}}$ siècle avaient lu la Métaphysique des mœurs et plus particulièrement la Doctrine du droit ? Le fait que Durkheim, agrégé de philosophie et non-juriste, soit à notre connaissance le seul à discuter à son époque en France la théorie kantienne sur les origines de la propriété ${ }^{33}$ nous laisse à penser (comme la très tardive pénétration de l'expression «autonomie de la volonté ») que nos professeurs de droit de la Belle Époque n'étaient pas vraiment des connaisseurs de Kant.

16 Partant des références expresses et des notes (ce qui implique de les vérifier, la vérification faisant apparaître des erreurs ou des citations de seconde main), en ayant la plus grande attention aux dates et aux diverses éditions, nous devons prolonger notre étude en tenant compte des recensions d'ouvrages étrangers (assez rares en France), des catalogues des bibliothèques, jusqu'à l'absence encore aujourd'hui de nombreux ouvrages juridiques étrangers $d u x{ }^{e}$ et $d u x^{e}$ siècle dans le fonds de la Bibliothèque nationale de France (ce qui est plutôt un indice de l'ignorance de ces ouvrages par les auteurs français des siècles passés). L'édition de traductions, ce que nous savons ou pouvons conjecturer de la connaissance des langues étrangères par les juristes du passé, le croisement de différentes circulations entre plusieurs pays (on ne peut par exemple se limiter à un « dialogue » franco-allemand, comme si les juristes de ces deux nations avaient entretenu un constant «tête à tête », ce qui n'est pas le cas) doivent contribuer à établir une chronologie plus fine de la pénétration des publications qui peuvent véhiculer des idées. Très souvent, ce type de recherches conduit à constater un décalage de plusieurs années, voire de plusieurs décennies, pour la réception des ouvrages étrangers (par exemple, la connaissance de Jhering parvient en France plutôt dans les années 1890 que dans les années 1860-1880 34) ou une véritable ignorance de pans entiers de la littérature étrangère. 
17 L'on retrouve ici la prégnance des cadres nationaux : des juristes formés dans leur pays d'origine à la lecture de la littérature nationale dans leur langue maternelle ne sont pas toujours bien informés des parutions à l'étranger de livres portant sur les sujets qu'ils traitent, même s'ils sont ouverts au comparatisme. L'ouvrage d'Hermann Isay, Die Isolierung des deutschen Rechtsdenkens paru à Berlin en 1924, en témoigne pour des juristes allemands pourtant à la pointe de la recherche scientifique au tournant des $\mathrm{xIx}^{\mathrm{e}}$ et $\mathrm{xx}^{\mathrm{e}}$ siècles ${ }^{35}$. Cet avocat, un des partisans tardifs du freie Recht sous la République de Weimar, se plaignait de l'isolement de la doctrine juridique allemande depuis la mise en vigueur du BGB et relevait notamment l'ignorance par les auteurs allemands des productions de Gény et Lambert comme de la littérature anglo-américaine. Il y a sans doute un peu d'excès dans ces reproches adressés par Isay à ses collègues allemands et autrichiens (Kelsen est expressément visé), mais aussi un jugement qui pourrait être généralisé à tous les pays : l'information dont dispose un juriste en matière d'idées est nécessairement limitée et le plus souvent déterminée par un contexte et des controverses de caractère national. Si les frontières se présentaient sous des formes diverses, avant ou après l'avènement des États modernes, avant ou après le développement tardif des passeports, avant ou après l'essor des grandes bibliothèques universitaires, avant ou après le déclin de l'usage du latin, ces frontières ont toujours rendu plus difficile l'accès aux livres venus de loin qu'aux livres produits à proximité. Aux difficultés d'accès aux publications étrangères s'ajoutent, comme on l'a dit, les obstacles à la compréhension de concepts ou de théories exogènes, une compréhension souvent dépendante de subtilités linguistiques et d'une bonne connaissance du contexte intellectuel dans lequel ces concepts et théories juridiques ont pris naissance dans un pays étranger.

18 Il y a également un autre obstacle de taille à l'histoire transnationale des idées juridiques qui tient au fait que les idées n'appartiennent à personne et que la présence d'idées analogues dans des espaces séparés (par la géographie mais aussi par le temps) ne signifie pas une influence des unes à l'égard des autres ou des phénomènes d'interaction. Les similitudes que relève Stefano Solimano, dans Verso il Code Napoleon ${ }^{36}$, entre les idées exprimées par Target dans son projet de Code civil et dans ses discours sur le droit pénal avec celles défen- 
dues à la même époque par Bentham et diffusées par quelques canaux en France permettent-elles d'induire une influence des théories de Bentham sur Target et plus généralement sur les codificateurs français ? Il est possible aussi de conclure à une ressemblance trompeuse ou à un parallélisme idéologique (ou morphologique par opposition à une influence généalogique) faisant que des idées comparables aient été proposées en Angleterre et en France, sans qu'on puisse parler d'antériorité ou même d'interactions ${ }^{37}$. L'histoire des idées nous a habitués, à tort ou à raison, à croire dans l'air du temps, dans des mouvements globaux et assez vagues (par exemple, en faveur de l'utilitarisme au début $\mathrm{du}_{\mathrm{xIx}}{ }^{\mathrm{e}}$ siècle) dont l'appréhension constitue un obstacle supplémentaire pour une étude minutieuse de la circulation des idées juridiques.

L'histoire transnationale des idées a ainsi tout à gagner de passer d'un point de vue général sur les mouvements d'idées à l'étude précise des flux de circulation, des impacts liés à l'utilisation de théories venues de l'étranger et des contextes (avec des effets de mode, comme l'anglophilie ${ }^{38}$ ou de rejet comme la germanophobie) dans lesquels ces emprunts sont acculturés dans le pays receveur. Dans la mesure où les idées n'ont pas à proprement parler de propriétaire, leur circulation n'obéit pas aux règles de l'importation et de l'exportation de produits ou de technologies, y compris de technologies juridiques. À la différence des énoncés juridiques qui peuvent être introduits par la force dans un ordre juridique étranger, notamment dans un contexte colonial ou semi-colonial, ou adoptés volontairement par un Gouvernement soucieux d'imiter tel ou tel modèle, les idées juridiques sont connues et discutées par des particuliers plutôt qu'imposées ou attirées d'un pays à un autre. Et les frontières qui sont susceptibles de ralentir la circulation des idées ne sont pas seulement territoriales, politiques ou linguistiques. Des idées peuvent avoir du mal à circuler en raison de leur caractère «exotique » ou hétérodoxe qui rend leur pénétration difficile chez des juristes habitués à des idées familières et « indigènes ». Le champ juridique, structuré nationalement au fur et à mesure que l'on avance dans le temps, n'est pas le plus propice à la circulation d'idées venues d'un autre champ juridique et auxquelles les juristes ne sont pas accoutumés en raison de leur éducation et de leur pratique. Une idée juridique, venue de l'étranger, peut rester méconnue ou sans effets, comme elle peut donner naissance à une autre 
idée, traduite et transformée, voire à un transplant juridique ${ }^{39}$. Les processus de circulation des idées juridiques sont variés, complexes, soumis à des aléas dont il faut reconnaitre l'importance si l'on veut dresser une cartographie des idées juridiques de caractère transnational.

\section{L'histoire transnationale des idées juridiques : à quelles fins?}

Les historien(ne)s du droit n'indiquent pas toujours, loin s'en faut, pourquoi ils pratiquent l'histoire transnationale des idées juridiques. À l'intérêt proprement historique pour la compréhension des débats d'idées entre juristes et des éléments transnationaux qui participent à ces débats, se mêle très souvent notre curiosité de chercheur pour les livres du passé qui nous éclairent sur la compréhension du droit, et cela sans nous soucier des frontières, en bénéficiant aujourd'hui d'un large accès à des livres étrangers par des publications en langue originelle ou en traduction. La perspective de théorie ou de philosophie du droit se combine ainsi avec l'étude historique. Il est tout à fait légitime d'utiliser les théoriciens du droit du passé, plus ou moins éloigné, à la fois pour comprendre les débats de leur temps, mais aussi pour éclairer des controverses antérieures ou postérieures. Par exemple, le recours aux idées et aux concepts de Kelsen peut être un moyen d'aborder l'histoire des idées juridiques sous la Révolution française ${ }^{40}$, voire pour des périodes plus anciennes ${ }^{41}$. Dans cette optique, nous classons les concepts, les théories et les écoles de pensée en fonction de nos perspectives contemporaines. Par exemple, nous allons considérer tel ou tel auteur comme positiviste ou jusnaturaliste, avec le risque de ne pas prendre en considération ce que cet auteur pouvait connaître de la littérature étrangère sur les thèses positivistes et jusnaturalistes, comme sur la manière de les nommer (nous avons vu que l'expression «positivisme juridique » est récente dans toutes les langues) et de les classer à l'époque étudiée. En faisant ce type de reconstructions a posteriori, nous risquons de perdre quelque peu le fil de la recherche proprement historique : celle qui établit, au cas par cas, la présence ou non de la circulation de travaux, puis les phénomènes d'acculturation ou de naturalisation des idées ayant leur point de départ exprimé à l'étranger et parvenues à la 
connaissance de nos juristes nationaux. Il faut, selon nous, instaurer ou du moins déclarer plus de distance entre notre analyse portée vers la théorie du droit et la compréhension historique des phénomènes de circulation des idées. Le temps des « grands récits », euxmêmes limités à une sélection d'auteurs (censés dialoguer entre eux à travers les siècles) mais couvrant de larges périodes avec une perspective téléologique ${ }^{42}$, est sans doute passé.

Lidée d'une généalogie transnationale des concepts juridiques est aussi fortement ébranlée par les réflexions actuelles sur l'étude des concepts juridiques ${ }^{43}$. Dans la mesure où beaucoup de concepts juridiques ont la caractéristique de se trouver présents à la fois dans les énoncés normatifs du droit et dans les opinions de la science du droit, leur circulation d'un espace linguistique est rarement simple, quand le nom du concept a un équivalent dans une langue étrangère et correspond à des règles de droit comparables ${ }^{44}$, ou plus complexe quand le nom du concept est « intraduisible », notamment parce qu'il correspond à des règles de droit qui sont ignorées dans un autre système (par exemple, le trust dans les pays de droit civil). Les concepts, dont on peut raffiner la typologie selon qu'ils sont désignés par des mots du vocabulaire courant, par des expressions propres à la langue du droit et définis par elle, qu'ils correspondent à des standards ou à des institutions (subsumant sous un même nom de concept plusieurs règles, par exemple en matière de mariage), peuvent être combinés dans ce qui constitue, notamment chez les «théoriciens du droit » (mais parfois dans les énoncés normatifs eux-mêmes), des théories comme l'abus de droit, la séparation des pouvoirs, la fraude à la loi... À ce niveau, chaque concepteur ou utilisateur d'une théorie en a sa propre définition, qu'elle soit explicite ou non. La circulation des théories juridiques est donc fortement personnalisée, attachée à tort ou à raison - comme pour Montesquieu avec la séparation des pouvoirs - à une référence individuelle. Paradoxalement, l'œuvre d'un juriste, particulièrement quand elle abondante et s'étale dans le temps, est une construction des lecteurs, qui en la recevant, lui assignent à travers le temps des caractères particuliers ${ }^{45}$. Ce qui circule est bien davantage l'image d'une ouvre que l'œuvre elle-même, rarement connue dans son intégralité des autres juristes, notamment hors de l'espace où elle s'est développée ${ }^{46}$. Parler de l'influence de Savigny, de Jhering, de Gény ou de Kelsen en dehors de leur pays d'origine sous 
la forme de grand courants d'idées qui seraient issus de ces auteurs comporte donc un risque de réductionnisme, si l'analyse n'est pas fondée sur une connaissance fine de la circulation transnationale des idées juridiques. Dans la mesure où les concepts ne s'engendrent pas les uns les autres ${ }^{47}$, mais se succèdent ou se superposent sans aucune logique, on peut comprendre « qu'ils n'ont pas d'histoire », au sens que Michel Troper a donné à cette phrase en apparence provocatrice $^{48}$. La présence des concepts à la fois dans le droit et dans la science du droit, comme les obstacles inhérents à leur traduction doivent nous rendre particulièrement circonspects relativement à une histoire transnationale des concepts qui risque d'essentialiser et de styliser des phénomènes complexes de transformation de ces concepts dans le temps et dans l'espace ${ }^{49}$. Les concepts juridiques ne sont pas des Idées (même ce qu'on appelle souvent en France des idées-force) qui évolueraient de manière autonome à travers l'histoire et voyageraient d'un pays à un autre, ce sont des produits d'une activité intellectuelle située qui donnent lieu à des lectures diverses.

L'histoire transnationale des idées a plutôt profit à multiplier les études ciblées et à travailler en termes de «moments », comme Frédéric Worms l'a proposé pour la philosophie française, avec beaucoup de prudence sur une supposée identité nationale qui expliquerait des caractères communs aux philosophes travaillant en France ${ }^{50}$. Réfléchir en termes de moments, c'est être attentifs aux contextes dans lesquels se situent les œuvres, aux véritables dialogues (mais aussi aux absences de communication) entre juristes de plusieurs pays à la même période. L'histoire de la réception des œuvres des juristes les plus prolifiques nous conduit à une réflexion sur la formation collective de courants d'idées ou d'écoles de pensée. Comme en matière d'idées politiques, ces mouvements collectifs d'idées (que l'on peut qualifier d'idéologies de manière neutre) peuvent être transnationaux, mais ils ne donnent pas lieu à la constitution d'organisations semblables aux partis ou aux confédérations de dimension internationale ${ }^{51}$. La compréhension de l'histoire transnationale des idées ne peut alors se séparer d'une sociologie des pratiques et des champs juridiques qui tient compte des espaces nationaux, de leur histoire et de leur configuration, comme des circulations plus ou moins intenses entre ces espaces. C'est dans cette pluralité de perspectives, ouvertes à la reconnaissance du caractère hybride des ordres juridiques, que 
peut prendre tout son sens une histoire transnationale des idées juridiques.

\section{NOTES}

1 P.-Y. Saunier, Transnational History, Londres, Palgrave Macmillan, 2013, p. 1-5 ; C. Maurel, Manuel d'histoire globale, Paris, Armand Colin, 2014, passim. Sur l'histoire connectée, S. Subrahmanyan, "Connected Histories : Notes towards a Reconfiguration of Early Modern Eurasia », Modern Asian Studies, 31/3, 1997, p. 735-762.

2 Pour un exemple récent, Th. Duve, « Entanglements in Legal History. Introductory Remarks », dans Th. Duve (ed.), Entanglements in Legal History: Conceptual Approaches, Francfort-sur-le-Main, Max Planck Institute for European Legal History, 2014, p. 3-25.

3 M. Villey, La formation de la pensée juridique moderne. Cours d'histoire de la philosophie du droit, Paris, Les Cours de droit, 1975 ; rééd. Paris, Presses universitaires de France, coll. «Quadrige manuels », 2013.

4 R. Daval, Histoire des idées en France, Paris, Presses universitaires de France, coll. «Que sais-je? », 1956.

5 R. Sacco, «Legal Formants : A Dynamic Approach to comparative Law », American Journal of Comparative Law, 1991, p. 1-34 et 343-401.

6 M. Angenot, L'histoire des idées, Liège, Presses universitaires de Liège, 2014, p. 61.

7 Par exemple, l'ouvrage de H. S. Jones, The French State in question. Public Law and Political Argument in the Third Republic, Cambridge, Cambridge University Press, 1993, ne nous paraît pas avoir eu l'impact qu'il méritait chez les spécialistes français de l'histoire des idées relatives au droit public.

8 M. Stolleis, Geschichte des öffentlichen Rechts in Deutschland, Munich, Beck, 4 vol., 1988, 1992, 1999, 2012 ; P. Grossi, Scienza giuridica italiana. Un profilo storico 1860-1950, Milan, Giuffrè, 2000 ; M. Lobban, A History of the Philosophy of Law in the Common Law World, 1600-1900, Dordrecht, Springer, 2007. Dans la même série, E. Pattaro (ed.), A Treatise of Legal Philosophy and General Jurisprudence, le volume de D. Canale, P. Grossi, H. Hofmann, A History of the Philosophy of Law in the Civil Law World 1600-1900, Dordrecht, Springer, 2009 s'inscrit aussi largement dans des cadres nationaux. 
Pour la France, Fr. Audren, J.-L. Halpérin, La culture juridique française. Entre mythes et réalités (XIX ${ }^{e}-\mathrm{xx}^{e}$ siècles), Paris, CNRS éd., 2013.

9 J.-L. Thireau, « La doctrine civiliste avant le Code civil », dans La doctrine juridique, Centre d'histoire du droit et de recherches normatives de Picardie, Paris, Presses universitaires de France, 1993, p. 40-43.

10 P. Goodrich, « Poor Illiterate Reason : History, Nationalism and Common Law », Social and Legal Studies, 1992/1, p. 7-28.

11 K. Luig, «The Institutes of National Law in the Seventeenth and Eighteenth Centuries », Juridical Review, 17, 1972, p. 193-226.

12 Sur les rapports entre historiens du droit britanniques, américains et allemands (laissant à l'écart leurs collègues français), D. Rabban, Law's History. American Legal Thought and the Turn to History, Cambridge, Cambridge University Press, 2009.

13 M. Koskenniemi, The Gentle Civilizer of Nations : The Rise of Fall of International Law, Cambridge, Cambridge University Press, 2002.

14 J.-L. Halpérin, Entre nationalisme juridique et communauté de droit, Paris, Presses universitaires de France, coll. «Les voies du droit », 1999, p. 115-116.

15 J.-L. Halpérin, « Vizioz », dans P. Arabeyre, J.-L. Halpérin, J. Krynen (dir.), Dictionnaire historique des juristes français $\mathrm{xII}^{e}-\mathrm{xx}^{e}$ siècle, Paris, Presses universitaires de France, $2^{\mathrm{e}}$ éd., 2015, p. 1009-1010.

16 J.-M. Denquin, « Présentation de Le pouvoir, l'ordre, la liberté et les erreurs des système subjectivistes », dans M. Touzeil-Divina (dir.), Maurice Hauriou, Miscellanées, Le Mans, Édition L'épitoge, 2014. p. 135 et s.

17 Le terme de concept renvoie à des définitions qui donnent à une ou plusieurs expressions linguistiques (des signifiants) la même signification ou signifié. De ce fait, les concepts correspondent à des noms, sachant qu'un même nom de concept peut être utilisé, par différents auteurs ou dans divers énoncés, pour des concepts qui sont en réalité dissemblables, leur définition n'étant pas identique. L'inverse peut évidemment se produire, un concept unique étant désigné selon les cas par plusieurs noms.

18 « Notice historique sur le chancelier Daguesseau », dans Euvres choisies du chancelier Daguesseau précédées d'une notice sur sa vie, Paris, Firmin Didot, 1877, p. 2.

19 H. Mohnhaupt, « Formen und Konkurrenzen juristischer Normativitäten im "Jus Commune" und in der Differentienliteratur (17./18. Jh.) », Rechtsgeschichte, 25, 2017, p. 115-126. 
20 Jhering a joué un rôle décisif dans cette diffusion du mot « norme » en définissant le droit comme la totalité (Inbegriff) des normes de contrainte (Zwangsnormen) en vigueur dans un État (R. $\mathrm{v}^{\mathrm{O}} \mathrm{n}$ Jhering, Der Zweck im Recht, Leipzig, Breitkopf \& Härtel, 1877, vol. I, p. 318). À la même époque, le pénaliste Karl Binding utilise également le mot de norme pour étudier les règles du droit pénal et leur violation (Die Normen und ihre Übertretung, 1872). Inspiré directement par Jhering, August Thon publie en 1878 un ouvrage intitulé Rechtsnorm und subjektives Recht qui s'ordonne autour de l'articulation entre le droit «objectif » des normes et les droits subjectifs des individus.

21 G. Filangieri, La scienza della legislazione, ed. a cura di V. Frosini, Rome, Istituto Poligrafico e Zecca dello Stato, 1984, vol. I, p. 49 ; L. Zupetta, Corso completo di legislazione penale comparata, Turin, Tipografia economica, 1852, p. 119.

22 Fr. Gény, Science et technique, vol. I, Paris, Sirey, 1914, p. 54 et 68-69.

23 Ibid., vol. II, Paris, Sirey, 1915, p. 22, 31 et 37.

24 À notre connaissance, Radbruch et Kelsen sont les premiers à utiliser l'expression «Positivismus » en théorie du droit à partir de 1914.

25 R. Savelli, Censori e giuristi : storie di libri, di idei e di costumi (secoli XVIXVII), Milan, Giuffrè, 2011 ; R. Birn, La censure royale des livres dans la France des Lumières, Paris, Odile Jacob, 2007.

26 M. Marion, Recherches sur les bibliothèques privées à Paris au milieu du xvIII ${ }^{e}$ siècle : 1750-1759, Paris Bibliothèque nationale, p. 143 sur la présence de Grotius dans les bibliothèques des robins ; R. Chartier, Lectures et lecteurs dans la France d'Ancien Régime, Paris, Le Seuil, 1989. À titre d'exemple sur la présence des livres de droit dans les bibliothèques des religieux, B. Dompnier, M.-H. Froeschlé-Chopard (dir.), Les Religieux et leurs livres à l'époque moderne, [Clermont-Ferrand], Presses universitaires Blaise Pascal, 2000 qui atteste la présence de Grotius dans ces bibliothèques.

27 D. Gilles, La pensée juridique de Jean Domat (1625-1696) : du Grand Siècle au Code civil, thèse dactylo. Aix-Marseille, 2004.

28 Sur le séjour de Hobbes à Paris et ses lettres, F. Tönnies, « Siebzehn Briefe des Thomas Hobbes an Samuel Sorbière », Archiv für Geschcihte der Philosophie, 1889, III, p. 58 et s.

29 M. Fumaroli, LẦge de l'éloquence, Genève, Droz, 1980, p. 406 et p. 557 (sur une rencontre entre l'avocat Bignon et Grotius). 
30 A. Le Gall, Pascal, Paris, Flammarion, 2000, p. 460.

31 Y. Glazou, Hobbes en France au XVIII siècle, Paris, Presses universitaires de France, 1993.

32 A. Bürge, Das französische Privatrecht im 19. Jahrhundert, Francfort-surle-Main, Klostermann, $2^{\mathrm{e}}$ éd., 1995, p. 43-50.

33 É. Durkheim, Leçons de sociologie, Paris, Presses universitaires de France, 2010, coll. «Quadrige », p. 161.

34 J. Whitman, «Jhering parmi les Français 1870-1918 », dans O. Beaud et P. Wachsmann (dir.), La science juridique française et la science juridique allemande de 1870 à 1918, Strasbourg, Presses universitaires de Strasbourg, 1997, p. 151-164. Grâce à son séjour d'études en Allemagne, Durkheim a été dès les années 1880 un bien meilleur connaisseur de la pensée de Jhering que la plupart des juristes français.

35 H. Isay, Die Isolierung des deutschen Rechtsdenken, Berlin, Franz Vahlen, 1924.

36 S. Solimano, Verso Il Code Napoleon. Il progetto di Codice civile di Guy Jean-Baptiste Target (1798-1799), Milan, Giuffrè, 1998.

37 X. Martin, « À propos d'un livre. Bentham, Target et le Code civil », Revue d'histoire des facultés de droit et de la science juridique, 20, 2000, p. 121-148.

38 É. Tillet, La Constitution anglaise, un modèle politique et institutionnel dans la France des Lumières, Aix-en-Provence, Presses universitaires d'AixMarseille, 2001.

39 Par exemple les idées de Pothier ont pu inspirer des décisions judiciaires dans les pays de common law : R. Zimmermann, The Law of Obligations, Oxford, Clarendon Press, 1990, p. 334-336.

40 M. Troper, «Sur l'usage des concepts juridiques en histoire », Annales, Économies, Sociétés, Civilisations, 47-6, 1992, p. 1171-1183.

41 N. Laurent-Bonne et X. Prévost (dir.), Penser l'ordre juridique médiéval et moderne, Paris, Lextenso, 2017.

42 Nous pensons à F. Wieacker, Privatrechtsgeschichte der Neuzeit, Tübingen, 2e éd., Vandenhoeck \& Ruprecht, 1967 (mêlant histoire des normes et histoire des idées juridiques en matière de droit privé, centré sur l'Allemagne, mais traitant aussi d'autres pays européens) et à M. Villey déjà cité. 
43 V. Champeil-Desplats, Méthodologies du droit et des sciences du droit, Paris, Dalloz, coll. « Méthodes du droit », p. 320-326.

44 R. von Jhering, Esprit du droit romain, trad. fr. O. de Meulenaere, Paris, A. Marescq aîné, vol. III, $\S 44$, p. 39 parle d'alphabet du droit pour ces concepts au caractère universel, tout en reconnaissant que ces concepts fonctionnent dans des configurations nationales déterminées par les droits positifs.

45 C'est ainsi que les spécialistes de l'histoire de la pensée juridique distinguent deux époques dans l'œuvre de Jhering ou plusieurs stades dans le développement des théories de Kelsen. Les contemporains de ces auteurs, lisant souvent une partie de leurs travaux, n'ont pas nécessairement distingué ces différentes phases.

46 Comme exemple, C. Petit «A Contributor to the Method of Investigation. Sobre la fortuna de Gény en America », Quaderni fiorentini per la storia del pensiero giurdico moderno, 20, 1991, p. 201-269.

47 Contrairement à ce qu'écrivait R. von Jhering, op. cit., vol. I, § 3, p. 40 (« les notions juridiques sont fécondes, elles s'accouplent et en engendrent d'autres $\gg)$.

48 M. Troper, Le droit et la nécessité, Paris, Presses universitaires de France, coll. «Léviathan », 2011, p. 262.

49 À l'instar de l'histoire des concepts de R. Koselleck, Begriffsgeschichten : Studien zur Semantik und Pragmatik der politischen und sozialen Sprache, Francfort-sur-le-Main, Suhrkamp, 2006.

50 F. Worms, La philosophie en France au $\mathrm{xx}^{e}$ siècle, Paris, Gallimard, coll. «Folio Essais », 2009, notamment p. 176-177, sur le risque de rabattre des caractères philosophiques communs sur un supposé caractère national, au détriment d'une recherche fine des filiations, des traditions, des dialogues explicites ou implicites.

51 Les mouvements d'idées en matière de droit international, donnant naissance à des associations porteuses de la science du droit international, sont l'exception qui confirme la règle comme l'ont montré les travaux de M. Koskenniemi, The Gentle Civilizer of Nations. The Rise and Fall of International Law 1870-1960, Cambridge, Cambridge University Press, 2001. 


\section{RÉSUMÉS}

\section{Français}

L'histoire transnationale peut-elle s'appliquer aux idées juridiques dans la mesure où celles-ci circulent d'un espace à un autre ? La réponse peut être positive, si l'on veut bien s'interroger sur les domaines appropriés de cette histoire transnationale, sur ses moyens et méthodes, enfin sur les fins poursuivies par les chercheurs.

\section{English}

Is transnational history available for legal ideas, that circulate from one space to another? The answer can be positive, if one accepts to think about the adequate fields of this transnational history, about the employed means and chosen methodologies, lastly about the targets pursued by the researchers.

\section{INDEX}

\section{Mots-clés}

histoire transnationale, circulations, concepts juridiques, grands récits, moments

Keywords

transnational history, circulations, legal concepts, great narratives, moments

\section{AUTEUR}

Jean-Louis Halpérin 